Brief Report

\title{
What Is the Estimated COVID-19 Reproduction Number and the Proportion of the Population That Needs to Be Immunized to Achieve Herd Immunity in Malaysia? A Mathematical Epidemiology Synthesis
}

\author{
Kurubaran Ganasegeran ${ }^{1, * \mathbb{D}}$, Alan Swee Hock Ch'ng ${ }^{1,2}$ and Irene Looi ${ }^{1,2}$ \\ 1 Clinical Research Center, Seberang Jaya Hospital, Ministry of Health Malaysia, Penang 13700, Malaysia; \\ alanchng1978@gmail.com (A.S.H.C.); looiirene@moh.gov.my (I.L.) \\ 2 Medical Department, Seberang Jaya Hospital, Penang 13700, Malaysia \\ * Correspondence: medkuru@yahoo.com or kurubaran@moh.gov.my
}

check for updates

Citation: Ganasegeran, K.; Ch'ng, A.S.H.; Looi, I. What Is the Estimated COVID-19 Reproduction Number and the Proportion of the Population That Needs to Be Immunized to Achieve Herd Immunity in Malaysia? A Mathematical Epidemiology Synthesis. COVID 2021, 1, 13-19. https://doi.org/10.3390/covid1010003

Academic Editor: Joan Puig-Barberà

Received: 2 March 2021

Accepted: 7 April 2021

Published: 1 June 2021

Publisher's Note: MDPI stays neutral with regard to jurisdictional claims in published maps and institutional affiliations.

Copyright: (c) 2021 by the authors. Licensee MDPI, Basel, Switzerland. This article is an open access article distributed under the terms and conditions of the Creative Commons Attribution (CC BY) license (https:/ / creativecommons.org/licenses/by/ $4.0 /)$.

\begin{abstract}
We aimed to determine Malaysia's COVID-19 reproduction number and herd immunity threshold through a mathematical epidemiology synthesis. Using time-series incidence data, the time-dependent reproduction number $\left(\mathrm{R}_{\mathrm{t}}\right)$ was yielded over time during the COVID-19 containment measures in Malaysia. The value of $R_{t}$ at the beginning of the epidemic and prior to any interventions in place was used to determine the proportion of the population that needs to be immunized to achieve herd immunity. $R_{t}$ was strongly influenced by interventions being put in place. We established that at least $74 \%$ of the Malaysian population needs to be vaccinated to achieve herd immunity against COVID-19. This threshold estimate is somewhat influenced by the availability of an efficacious vaccine. A vaccine with $95 \%$ efficacy would approximately synthesize a herd immunity threshold of $78 \%$. We conclude that $\mathrm{R}_{\mathrm{t}}$ is a valid estimator to determine the effectiveness of control measures and a parameter of use to synthesize herd immunity thresholds in the current COVID-19 pandemic.
\end{abstract}

Keywords: COVID-19; reproduction number; herd immunity; vaccine; Malaysia

\section{Introduction}

The ultimate challenge faced by public health workers during infectious disease outbreaks is to implement rapidly effective control measures that have the capacity to break the chain of transmission and to establish a population's herd immunity. Mathematical algorithms and models have been used to guide public health workers to assess the optimization of control strategies for subsequent decision-making. However, the parameters used to yield statistical estimates are highly dependent on the availability of surveillance data and temporal changes of disease patterns. The fundamental value to synthesize the anticipated mathematical models for further interventions is dependent on the estimated reproduction number.

The evolution of infectious disease dynamics has applied four principal strategies to yield reproduction numbers to date, namely the basic reproduction number $\left(R_{0}\right)[1]$, the effective reproduction number $(R)$ [2], the instantaneous reproduction number [3], or its extension derivative, the time-varying reproduction number $\left(R_{t}\right)[4]$. While the primer $\left(\mathrm{R}_{0}\right)$ was contextualized as the number of secondary cases generated by the presence of an index case in an otherwise susceptible, well-mixed population [1], $R$ on the other hand was postulated to be a more useful estimate during an epidemic spread, as it takes into account the actual average number of secondary cases that will arise from an index case. If $R<R_{0}$, it is highly possible to deduce that the implemented interventions for epidemic control were effective [2]. However, $R$ only estimates the average number of secondary cases from an index case at time $t$, while not being "instantaneous" beyond time $t$, even if interventions 
or control measures are being put in place or removed [4]. To overcome this limitation, an "instantaneous" reproduction number $\left(\mathrm{R}_{\mathrm{t}}\right)$ that synthesizes transmissibility over time has been advocated for by Cori and colleagues [3]. While this framework is widely used, it suffers from two critical limitations: the first is the requirement to have a serial interval estimation, which is unlikely to be available at the early stage of an epidemic, while the second is the inability to distinguish total incident cases from that of imported ones, which has the tendency to cause overestimation [3,4]. The recent version of $R_{t}$ as proposed by Thompson and colleagues [4] is an extended framework by Cori and colleagues [3] that has the capacity to offset these limitations.

As $R_{t}$ is real-time, it has multiple advantages over $R_{0}$. Firstly, $R_{t}$ is a time-dependent estimate that is capable to assess if implemented control measures are effective to contain the outbreak, or whether additional interventions are required over time. Secondly, as $R_{t}$ represents the expected number of secondary cases that arise from an index case at time $t$, the value of the estimate changes over time throughout the outbreak. If $R_{t}<1$, the outbreak will likely be controlled, but if $R_{t}>1$, a sustained infection rate is likely. The principal aim of control measures is to reduce $R_{t}$ to be lower than one. Thirdly, with $R_{t}$, it is possible to estimate the minimum proportion of the population that needs to be immunized to achieve herd immunity, with the assumption that the value of $R_{t}$ is yielded at the time prior to interventions being put in place. This could be calculated using the formula $P_{\text {crit }}=1-\left(1 / R_{t}\right)$ as advocated by Anderson and May [1] and tested by Kwok and colleagues [5] for the recent COVID-19 pandemic, in which $P_{\text {crit }}$ represents the minimal ("critical") level of population's immunity that could be acquired via vaccination. However, this assumption is only valid for specified situations as $R_{t} \neq R_{0}$. The classical formula for calculating herd immunity is $1-\left(1 / R_{0}\right)$. $R_{t}$ will only equal to $R_{0}$ with the assumption that the whole population is susceptible [1,5], and the valid estimator of $R_{t}$ is the parameter yielded prior to any interventions being in place or at the beginning of the epidemic.

Herd immunity is conceptualized as the proportion of the population that needs to become immune to an infectious disease outbreak so that those without immunity are unlikely to interact with an infected person and become infected. To establish herd immunity, epidemiologists first need to know how contagious the infection is, and concurrently be able to determine how many nonimmune people are susceptible to acquiring the infection from an infected person; in other words, what is the reproduction number yielded in a particular population being infected with the outbreak? Typically, herd immunity thresholds vary between populations or countries, based on varying infectiousness of the disease, which is highly dependent on accuracies of diagnostic tests used to detect cases, testing capacities, host characteristics, and environmental risk factors that influence the dynamics of disease transmission [6,7].

The herd immunity threshold is somewhat influenced by the proportional vaccine efficacy and the desirable efficacious value $(\varepsilon)$ for any vaccine is $80 \%$. Malaysia has acquired a vaccine deal that is claimed to be $95 \%$ efficacious, and is projected to be available for the Malaysian population in the first quarter of 2021 [8]. With this proportional vaccine efficacy, the extended formula to calculate the threshold of herd immunity could be expressed by the formula $P_{\text {crit }}=1-\left(1 / R_{t}\right) / \varepsilon$ [9]. Here, we applied a mathematical epidemiology synthesis approach to determine the trends of $\mathrm{R}_{t}$ and the minimal "critical" level ( $\mathrm{P}_{\text {crit }}$ ) of the Malaysian population's immunity threshold for COVID-19.

\section{Materials and Methods}

\subsection{Data Source and Tools}

Using the global multi-country COVID-19 statistics estimate from Our World in Data (dataset available from https: / / ourworldindata.org/coronavirus, accessed on 1 December 2020) [10], which was subsequently checked for consistency with two local real-time data captures, namely from outbreak.my (accessible at https: / /www.outbreak.my /, accessed on 1 December 2020) and the Ministry of Health Malaysia COVID-19 current situational pandemic data capture (accessible at: http:/ / covid-19.moh.gov.my/, accessed on 1 De- 
cember 2020), we synthesized $R_{t}$ using an epidemiological tool, EpiEstim App (accessible at https: / / shiny.dide.imperial.ac.uk/epiestim, accessed on 5 December 2020), which is also available as an R package (EpiEstim 2.2). Both yielded consistent, valid, and reliable estimates [3,4].

\subsection{Model Assumptions of $R_{t}$}

Based on the approach advocated and tested by EpiEstim [3,4,11], we assumed that the total numbers of incident COVID-19 cases arising from timestep $t$, denoted as $I_{t}$, will be the summation of numbers that constitute both local $\left(I_{t}^{\text {local }}\right)$ and imported $\left(I_{t}^{\text {imported }}\right)$ COVID-19 cases in Malaysia, thus yielding the following equation:

$$
I_{t}=I_{t}^{\text {local }}+I_{t}^{\text {imported }}
$$

$R_{t}$ is principally contextualized as the ratio between new locally infected cases at time $t$, $I_{t}^{\text {local }}$, with the total infectivity potential of all infected individuals at time $t$ denoted as $\Lambda_{\mathrm{t}}$. When there is a presence of a single serial interval distribution, $\omega_{\mathrm{s}}(\mathrm{s}=1,2,3,4,5, \ldots)$ which simply denotes the probability of a secondary case surfaced at time $s$ after the index case, the incident case (either local or imported) that appears in the previous time-step, $t-s$, is assumed to contribute to its latest infectiousness at a level relative to $\omega_{\mathrm{s}}$. Being conditional on $\omega_{\mathrm{s}}, \Lambda_{\mathrm{t}}$ yields the following equation:

$$
\Lambda_{\mathrm{t}}\left(\omega_{\mathrm{s}}\right)=\sum_{\mathrm{s}=1}^{\mathrm{t}} \mathrm{I}_{\mathrm{t}-\mathrm{s}} \omega_{\mathrm{s}}
$$

The likelihood of incidence data (seen as a Poisson count) observed over a sliding time window of size $\tau$ that ends at $t$ is maximized. It is assumed that the reproduction number is constant throughout this sliding time window $(t-\tau, t)$. Hence, estimation of the reproduction number at each time window, denoted as $R_{t}, \tau$, for the time interval $(t-\tau, t)$ is verified by the equation:

$$
\mathrm{R}_{\mathrm{t}, \tau}(t)=\underset{\mathrm{Rt}}{\operatorname{argmax}} \prod_{k=t-\tau}^{t} \frac{\left(\mathrm{R}_{\mathrm{t}} \Lambda_{k}\left(\omega_{s}\right)\right)^{I_{k}} \exp \left(-\mathrm{R}_{\mathrm{t}} \Lambda_{k}\left(\omega_{s}\right)\right)}{I_{k} !}
$$

The $R_{t}$ is conceptually yielded from both incidence and available serial interval data, thereby computing relevant mean and $95 \%$ credible intervals. The synthesis of $R_{t}$ is based on underlying assumptions as follows:

I. The COVID-19 epidemic would be ongoing, and to evaluate the effectiveness of interventions, the total number of infections caused by the latest detected cases are yet to be known.

II. The calculations are based on the potential number of secondary infected persons that a cohort of cases could have caused if the transmissibility pattern had remained the same at the time of their detection.

III. Local cases are distinguished from imported cases.

\subsection{Analytical Procedure}

Daily incidence of "all cases" and a separate dataset that distinguished imported cases (retrieved from Malaysia's daily situational update available from http:/ / covid19.moh.gov.my/, accessed on 1 December 2020) were analyzed concurrently to avoid overestimation [4]. Selecting the sliding time window of fourteen days (given the upper limit of COVID-19 incubation period), for which $R_{t}$ was to be estimated, we chose a distributional estimate of serial interval for parametric without uncertainty (an offset gamma), being a conjugate to Poisson likelihood) through a Bayesian framework to yield the $R_{t}$ over time, with estimated mean and $95 \%$ credible intervals to be synthesized. The yielded $R_{t}$ prior to any interventions executed in Malaysia, with the assumption that the 
whole population was susceptible at the beginning of the COVID-19 outbreak in Malaysia, was subsequently used to determine $\mathrm{P}_{\text {crit }}$.

\subsection{COVID-19 Interventions}

The COVID-19 control measures executed in Malaysia were principally classified into three phases: the Movement Control Order (MCO), the Conditional Movement Control Order (CMCO), and the Recovery Movement Control Order (RMCO). The MCO was enacted on 18 March 2020 with full enforcement using legislative measures. The CMCO was enforced on 4 May 2020 with soft reopening of the economic and business sectors, but subjected to strict Standard Operating Procedures (SOPs). The RMCO was implemented from 10 June 2020 with gradual reopening of social, economic, and educational activities under strict SOPs, with the exception that international borders remained closed. All phases mandated high compliance to non-pharmacological measures, such as physical distancing and masking [12]. To appreciate if trends of reproduction numbers were influenced by interventions being put in place, we sub-grouped yielded $R_{t}{ }^{\prime} s$ overtime into four major phases, namely pre-MCO (non-intervention phase), MCO, CMCO, and RMCO.

\section{Results}

\subsection{Estimated Reproduction Numbers $\left(R_{t}\right)$}

Table 1 exhibits $R_{t}$ values over time with interventions being put in place. The mean (95\% credible intervals) $R_{t}$ fourteen days prior to MCO was $3.91(2.69,5.35)$. During interventions, there were consequent decreases of $R_{t}$, from $2.52(2.34,2.70)$ from the first phase of MCO, to a subsequent $R_{t}$ of $1.12(1.07,1.17)$ at MCO phase $2, R_{t}$ of $0.97(0.93,1.01)$ at MCO phase 3 and $\mathrm{R}_{\mathrm{t}}$ of $0.59(0.55,0.64)$ at MCO phase 4 . At the CMCO and RMCO phases, $R_{t}$ showed inconsistencies with periodic upward and downward trends (Table 1).

Table 1. Cumulative number of positive cases $(\mathrm{N})$ and estimated time-dependent reproductive number $\left(\mathrm{R}_{\mathrm{t}}\right)$ of an ongoing epidemic.

\begin{tabular}{|c|c|c|c|}
\hline Phase & Duration & $\mathbf{N}$ & $R_{t}(95 \%$ Credible Intervals) \\
\hline \multirow{4}{*}{ Pre-MCO } & $1 / 22 / 2020-2 / 4 / 2020$ & 10 & $0.19(0.02,0.54)$ \\
\hline & $2 / 5 / 2020-2 / 18 / 2020$ & 22 & $0.15(0.02,0.43)$ \\
\hline & $2 / 19 / 2020-3 / 3 / 2020$ & 36 & $0.16(0.02,0.46)$ \\
\hline & $3 / 4 / 2020-3 / 17 / 2020$ & 673 & $3.91(2.69,5.35)$ \\
\hline \multirow{4}{*}{$\mathrm{MCO}$} & $3 / 18 / 2020-3 / 31 / 2020$ & 2766 & $2.52(2.34,2.70)$ \\
\hline & $4 / 1 / 2020-4 / 14 / 2020$ & 4987 & $1.12(1.07,1.17)$ \\
\hline & $4 / 15 / 2020-4 / 28 / 2020$ & 5851 & $0.97(0.93,1.01)$ \\
\hline & $4 / 29 / 2020-5 / 12 / 2020$ & 6742 & $0.59(0.55,0.64)$ \\
\hline \multirow{2}{*}{$\mathrm{CMCO}$} & $5 / 13 / 2020-5 / 26 / 2020$ & 7604 & $0.80(0.74,0.86)$ \\
\hline & $5 / 27 / 2020-6 / 10 / 2020$ & 8338 & $1.16(1.08,1.24)$ \\
\hline \multirow{11}{*}{$\mathrm{RMCO}$} & $6 / 11 / 2020-6 / 24 / 2020$ & 8596 & $0.62(0.57,0.67)$ \\
\hline & $6 / 25 / 2020-7 / 8 / 2020$ & 8677 & $0.62(0.53,0.69)$ \\
\hline & $7 / 9 / 2020-7 / 22 / 2020$ & 8831 & $0.36(0.25,0.48)$ \\
\hline & $7 / 23 / 2020-8 / 5 / 2020$ & 9023 & $0.78(0.62,0.95)$ \\
\hline & $8 / 6 / 2020-8 / 19 / 2020$ & 9235 & $0.58(0.48,0.69)$ \\
\hline & $8 / 20 / 2020-9 / 2 / 2020$ & 9360 & $0.66(0.55,0.78)$ \\
\hline & $9 / 3 / 2020-9 / 16 / 2020$ & 10,031 & $1.27(1.17,1.37)$ \\
\hline & $9 / 17 / 2020-9 / 30 / 2020$ & 11,224 & $1.36(1.29,1.44)$ \\
\hline & $10 / 1 / 2020-10 / 14 / 2020$ & 17,540 & $1.49(1.46,1.53)$ \\
\hline & $10 / 15 / 2020-10 / 28 / 2020$ & 29,441 & $1.16(1.14,1.18)$ \\
\hline & $10 / 29 / 2020-11 / 11 / 2020$ & 42,872 & $1.03(1.01,1.05)$ \\
\hline CMCO & $11 / 12 / 2020-11 / 25 / 2020$ & 59,817 & $1.14(1.12,1.15)$ \\
\hline
\end{tabular}

Note: Estimates are based on fourteen days interval in view of COVID-19 incubation period; MCO-Movement Control Order; CMCOConditional Movement Control Order; RMCO-Recovery Movement Control Order; $\mathrm{R}_{\mathrm{t}}$ was predicted using a Bayesian framework, with estimated serial interval (mean $=4.7$ days, $\mathrm{SD}=2.9$ days) as proposed by Nishiura et al. [13]; time-series incidence data were available up to 25 November 2020. 


\subsection{Critical Level $\left(P_{\text {crit }}\right)$ of the Malaysian Population's Herd Immunity Threshold}

The minimum expected proportion of the population that needs to be immunized to achieve herd immunity using the formula $P_{\text {crit }}=1-\left(1 / R_{t}\right)$ was $74 \%$. If we assume that the projected deal of the vaccine acquirement by Malaysia in the first quarter of 2021 materializes and that the vaccine is $95 \%$ efficacious, hence using the extended valid formula of $P_{\text {crit }}=1-\left(1 / R_{t}\right) / \varepsilon$, the estimated herd immunity threshold for the Malaysian population will be $78 \%$. Lower efficacious vaccine values would synthesize higher thresholds for the population's herd immunity (Table 2).

Table 2. Vaccine efficacy $(\varepsilon)$ and estimated herd immunity threshold $\left(\mathrm{P}_{\text {crit }}\right)$.

\begin{tabular}{cc}
\hline Vaccine Efficacy $(\varepsilon)$ & Population's Herd Immunity Threshold $\left(\mathbf{P}_{\text {crit }}\right)$ \\
\hline $95 \%$ & 0.78 \\
$90 \%$ & 0.82 \\
$85 \%$ & 0.88 \\
$80 \%$ & 0.93 \\
\hline
\end{tabular}

\section{Discussion}

The current paper has shown that while public health systems worldwide struggle to contain the spread of the COVID-19 pandemic through rapid implementation of legislative and non-pharmacological interventions, the effectiveness of such measures to break the chain of infection or to establish a population's herd immunity could be evaluated through mathematical algorithms. The yielded $\mathrm{R}_{\mathrm{t}}$ parameters over time for the current mathematical epidemiology synthesis in Malaysia has observed either a declining or increasing trend over time. The $\mathrm{R}_{\mathrm{t}}$ value yielded fourteen days prior to $\mathrm{MCO}$ was above three, consistent with the report by the Ministry of Health Malaysia [14]. A plausible explanation that could be advocated here is that the strength of implemented interventions, such as both legislative and community mitigation measures with high compliance to SOPs, would have influenced $R_{t}$ trends. Such trends were observed by Li and colleagues [15].

Through the Prevention and Control of Infectious Diseases Act 1988 and the Police Act 1967, the MCO in Malaysia prohibited mass movements, religious gatherings, sports, social or cultural activities, and enforced closures of business premises [12]. Self-quarantine was made compulsory from those returning abroad and travel restrictions were imposed on tourists and visitors; kindergartens, schools, and universities were closed in addition to government and private premises, except for essential services. In contrast, the CMCO allowed the soft reopening of the economic and business sector with the exception that these sectors will not risk infecting people. The educational and social sectors still remained closed. In addition to legislative measures, the compliance of the implemented SOPs based on avoidance of the 3Cs (Crowded places, Confined places, Closed conversation) and the practice of $3 \mathrm{Ws}$ (Wash hands, Wear masks, Warn against risks) were highly promoted [12,16]. As Malaysia observed a depleting number of cases, the RMCO was implemented in June 2020, facilitating full reopening of the economic and social sectors, with the exception that borders remained closed and non-pharmacological interventions are highly adhered to [16]. Such measures were crucial as the nation needed to equilibrize between lives and livelihoods of the people, preventing unprecedented economic and social impacts to the country. However, over time there were periodic emergences of new clusters, observing an exponential rise of new cases due to non-adherence to SOPs, that subsequently forced the government to revert back to the CMCO stage from November 2020 [17]. This phenomenon could be attributed to the inconsistent trends of yielded $R_{t}$ values at the $\mathrm{CMCO}$ and RMCO phases.

The herd immunity threshold of $78 \%$ (if a vaccine with $95 \%$ efficacy becomes available now) for the total Malaysian population will be a valid, reliable, and practical estimate to be of most use, as it assumes the proportion of the population that needs to be immune before life could return to "pre-COVID-19 normal". The yielded estimate is within the anticipated epidemiological range of $70-90 \%$ to achieve a population's herd immunity [6]. 
This mathematical epidemiology synthesis could be a fundamental guide for Malaysia to plan appropriate distribution of vaccines for COVID-19 when they become available, with the aim to establish herd immunity through spatial epidemiological COVID-19 hotspot analysis, promising a resource for the planning of cost-saving measures for the government and health facilities nationwide.

\section{Limitations}

Limitations of this mathematical epidemiology synthesis should be acknowledged. Firstly, although the efficacy of the current COVID-19 vaccines is known, their effectiveness (duration of protection) are yet to be established. We anticipate that the time required to vaccinate $78 \%$ of the Malaysian population (more than 25 million people) may extend to over a year, and if vaccine effectiveness is shorter than expected, this could compromise the establishment of herd immunity. Secondly, the current synthesis excluded the percentage of the population that survived natural infection (considered immune for the next exposure) with symptoms. Thirdly, the actual number of infected cases in Malaysia is not known. The actual number of cases may reach up to 8 times or more as compared to tested positive cases based on the Center for Disease Control (CDC) simple probabilistic multiplier model estimation [18], as certain adults and most children under 16 years of age usually do not show symptoms after infection. Fourthly, the demographic structure of the Malaysian population such as different age groups and geographic variations was neglected while estimating $R_{t}$ and herd immunity thresholds. Finally, although the $R_{t}$ metric can be used as a near real-time indicator to assess the effectiveness of interventions, the delays between infection and case observation data (which is unavoidable in most surveillance systems) may pose challenges to estimate $R_{t}$ in the near real-time, and to accurately infer the timing of $R_{t}$ movement. However, by generating synthetic data from deterministic and stochastic models, Gostic and colleagues [19] strongly recommended the use of EpiEstim model for analysis, as used in the current paper, due to its best fit for synthesizing real-time estimates of $R_{t}$ values with minimal bias, of both systematic over- or underestimations, and temporal inaccuracies. Thus, our estimates, although valid and reliable, need to be interpreted with caution after taking these limitations into consideration.

\section{Conclusions}

The synthesized $R_{t}$ values clearly show that the parameter of a valid estimate was strongly influenced by the effectiveness and adherence to interventions over time. Populationlevel interventions that Malaysia executed in response to the COVID-19 outbreak have shown real-time effects on interrupting the overall transmission dynamics. As Malaysia needs to balance between economic activity and disease transmission, control measures that were implemented in four major phases substantially relied on real-time assessment of $R_{t} s$, allowing public health professionals to tune relaxation decisions by ensuring that the infectiousness of COVID-19 is significantly reduced or plateaued, yet sustaining it to be less than 1 . We conclude that the minimum proportion of the Malaysian population to be immunized with a vaccine of $95 \%$ efficacy would be $78 \%$, in the quest to achieve the population's herd immunity. Lower vaccine efficacy values would yield higher herd immunity thresholds.

Author Contributions: Conceptualization, K.G., A.S.H.C. and I.L.; methodology, K.G.; formal analysis, K.G.; investigation, K.G., A.S.H.C. and I.L.; writing—original draft preparation, K.G.; writingreview and editing, A.S.H.C.; supervision, I.L. All authors have read and agreed to the published version of the manuscript.

Funding: This research received no external funding.

Institutional Review Board Statement: Not applicable.

Informed Consent Statement: Not applicable. 
Data Availability Statement: Publicly available datasets were analyzed in this study. This data can be found here: https:/ / ourworldindata.org/coronavirus (accessed on 1 December 2020); https: / / www.outbreak.my / (accessed on 1 December 2020); http:/ / covid-19.moh.gov.my/ (accessed on 1 December 2020).

Acknowledgments: We would like to thank the Director General of Health Malaysia for the support and permission to publish this article.

Conflicts of Interest: The authors declare no conflict of interest.

\section{References}

1. Anderson, R.M.; May, R.M. Infectious Diseases of Humans: Dynamics and Control; Oxford Science Publications: Oxford, UK, 1992; p. 768. ISBN 9780198540403.

2. Wallinga, J.; Teunis, P. Different epidemic curves for severe acute respiratory syndrome reveal similar impacts of control measures. Am. J. Epidemiol. 2004, 160, 509-516. [CrossRef] [PubMed]

3. Cori, A.; Ferguson, N.M.; Fraser, C.; Cauchemez, S. A new framework and software to estimate time-varying reproduction numbers during epidemics. Am. J. Epidemiol. 2013, 178, 1505-1512. [CrossRef] [PubMed]

4. Thompson, R.N.; Stockwin, J.E.; van Gaalen, R.D.; Polonsky, J.A.; Kamvar, Z.N.; Demarsh, P.A.; Dahlqwist, E.; Li, S.; Miguel, E.; Jombart, T.; et al. Improved inference of time-varying reproduction numbers during infectious disease outbreaks. Epidemics 2019, 29, 100356. [CrossRef] [PubMed]

5. Kwok, K.O.; Lai, F.; Wei, W.I.; Wong, S.Y.S.; Tang, J.W.T. Herd immunity-Estimating the level required to halt the COVID-19 epidemics in affected countries. J. Infect. 2020, 80, e32-e33. [CrossRef] [PubMed]

6. Rubin, R. Difficult to determine herd immunity threshold for COVID-19. JAMA 2020, 324, 732. [CrossRef] [PubMed]

7. Fontanet, A.; Caucemez, S. COVID-19 herd immunity: Where are we? Nat. Rev. Immunol. 2020, 20, 583-584. [CrossRef] [PubMed]

8. Mutalib, Z.A. Acquisition of Pfizer Covid-19 Vaccine Hinges on FDA, NPRA Approval. Available online: https://www.nst.com. $\mathrm{my} /$ news/nation/2020/11/645157/acquisition-pfizer-covid-19-vaccine-hinges-fda-npra-approval (accessed on 30 December 2020).

9. Anderson, R.M.; Vegvari, C.; Truscott, J.; Collyer, B.S. Challenges in creating herd immunity to SARS-CoV-2 infection by mass vaccination. Lancet 2020, 396, 1614-1616. [CrossRef]

10. Roser, M.; Ritchie, H.; Ortiz-Ospina, E.; Hasell, J. Coronavirus Pandemic (COVID-19). Available online: https:/ / ourworldindata. org/coronavirus (accessed on 1 December 2020).

11. Talmoudi, K.; Safer, M.; Letaief, H.; Hchaichi, A.; Harizi, C.; Dhaouadi, S.; Derouiche, S.; Bouaziz, I.; Gharbi, D.; Najar, N.; et al Estimating transmission dynamics and serial interval of the first wave of COVID-19 infections under different control measures: A statistical analysis in Tunisia from February 29 to May 5, 2020. BMC Infect. Dis. 2020, 20, 914. [CrossRef] [PubMed]

12. Ganasegeran, K.; Ch'ng, A.S.H.; Looi, I. COVID-19 in Malaysia: Crucial measures in critical times. J. Glob. Health 2020, 10, 020333. [CrossRef] [PubMed]

13. Nishiura, H.; Linton, N.M.; Akhmetzhanov, A.R. Serial interval of novel coronavirus (COVID-19) infections. Int. J. Infect. Dis. 2020, 93, 284-286. [CrossRef] [PubMed]

14. CodeBlue. MCO Slashed Malaysia Covid-19 Infection Rate by Over Three Times. Available online: https://codeblue.galencentre org /2020/04/10/mco-slashed-malaysia-covid-19-infection-rate-by-over-three-times/ (accessed on 30 December 2020).

15. Li, Y.; Campbell, H.; Kulkarni, D.; Harpur, A.; Nundy, M.; Wang, X.; Nair, H. The temporal association of introducing and lifting non-pharmaceutical interventions with the time-varying reproduction number (R) of SARS-CoV-2: A modelling study across 131 countries. Lancet Infect. Dis. 2021, 2, 193-202. [CrossRef]

16. Rampal, L. COVID-19 pandemic update. Med. J. Malays. 2020, 75, 195-198.

17. Rampal, L.; Liew, B.S.; Choolani, M.; Ganasegeran, K.; Pramanick, A.; Vallibhakara, S.A.; Tejativaddhana, P.; Hoe, V.C. Battling COVID-19 pandemic waves in six South-East Asian countries: A real-time consensus review. Med. J. Malays. 2020, 75, 613-625.

18. Reese, H.; Iuliano, A.D.; Patel, N.N.; Garg, S.; Kim, L.; Silk, B.J.; Hall, A.J.; Fry, A.; Reed, C. Estimated incidence of coronavirus disease 2019 (COVID-19) illness and hospitalization-United States, February-September 2020. Clin. Infect. Dis. 2020, ciaa1780. [CrossRef] [PubMed]

19. Gostic, K.M.; McGough, L.; Baskerville, E.B.; Abbott, S.; Joshi, K.; Tedijanto, C.; Kahn, R.; Niehus, R.; Hay, J.A.; De Salazar, P.M.; et al. Practical considerations for measuring the effective reproductive number, $\mathrm{R}_{\mathrm{t} .}$ PLoS Comput. Biol. 2020, 16, e1008409. [CrossRef] [PubMed] 